\title{
Synthesis of adenine-cyclen conjugates
}

\author{
Chuan-Qin Xia, Liang-Bo Zhu, Xing-Yu Tan, Yang Yue, and Xiao-Qi Yu* \\ Department of Chemistry, Key Laboratory of Green Chemistry and Technology (Ministry of \\ Education), Sichuan University, Chengdu 610064, China \\ E-mail:xqyu@tfol.com
}

(received 27 Jun 05; accepted 07 Sep 05; published on the web 09 Sep 05)

\begin{abstract}
A synthetic route to novel conjugates of adenine with cyclen was described in this paper and the target products were obtained in high yields. The spacer connecting cyclen and adenine is a rigid phenyl group. Preliminary results demonstrated that the adenine-cyclen conjugates can bind $\mathrm{Zn}^{2+}$ rapidly in water.
\end{abstract}

Keywords: Adenine, cyclen, conjugates, synthesis

\section{Introduction}

With the growth in the field of supramolecular chemistry and consequently a better understanding of how molecules interact with each other, more and more information is emerging on the complex supramolecular behaviour of nucleobases as supramolecular motifs. ${ }^{1-5}$ There are two major nucleobase binding motifs present in nucleic acids, adenine-thymine, AT (or adenine-uracil, AU in RNA) and guanine-cytosine, GC. While the selectivity of this base-pair interaction is controlled mainly by hydrogen bonding, both $\pi$ - $\pi$ stacking and hydrophobic effects also play a role in stabilizing the resulting structure. Utilization of the common nucleobases in supramolecular chemistry offers the flexibility of exploiting four different binding units $A, C, G$, and $\mathrm{T}$ (or $\mathrm{U}$ ), all of which offer different binding characteristics. Just because of this reason, the nucleobase as supramolecular motif has attracted much interest, resulting in much reseach in this area. $^{6-8}$

Cyclen (1, 4, 7, 10-tetraaza cyclododecane) possesses a strong coordination ability towards a wide range of cations, including transition metal ions and lanthanide ions, 9,10 and their complexes have been widely used as MRI contrast agents, ${ }^{11,12}$ luminescent probes, ${ }^{12,13}$ DNA recognition, ${ }^{14,15}$ DNA cleavers, ${ }^{16}$ enzyme mimics, ${ }^{17,18}$ and medicines for radioimmunotherapy. ${ }^{19,20}$ Yet, cyclen appended with a nucleobase through different spacers, which have large affinity constants for transition metal ions, are apparently rare. 
In this paper we synthesized two conjugates of adenine-cyclen. The building blocks are a particularly interesting target because: (I) conjugates of adenine-cyclen can form stable complexes with different metal ions so that binding might be important for supramolecular recognition, especially for selectively recognizing uracil in nucleic acids. ${ }^{21}$ (II) We choose the phenyl group as spacer in order to strengthen the recognition ability because of its $\pi$ - $\pi$ stacking possibility. (III) The conjugates of adenine-cyclen indicate that this work can be extended to design receptors for the site-specific binding of nucleic acids. To the best of our knowledge, this is the first example of adenine-cyclen conjugates.

\section{Results and Discussion}

The synthetic route of cyclen which was prepared by a previously reported method ${ }^{22}$ is shown in Scheme 1.

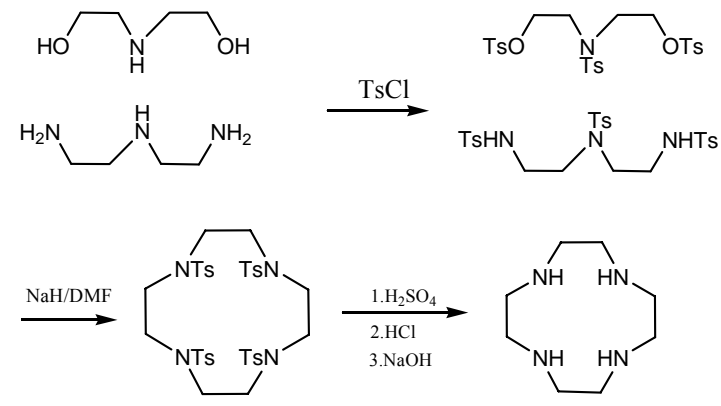

\section{Scheme 1}

The synthesis of adenine-cyclen conjugates can be performed using two main strategies. The direct $\mathrm{N}$-alkylation of adenine was first chosen to synthesize the required product $\mathbf{6}$ as described in Scheme 2. Unfortunately, with this method the reaction of compound $\mathbf{1}$ and $\mathbf{2}$ only produced $\mathbf{3}$ in $20 \%$ yield due to the multiple reactive centers of $\mathbf{1}$. The method proved so difficult that we did not perform the synthesis of compound $\mathbf{6}$ using this synthetic route.
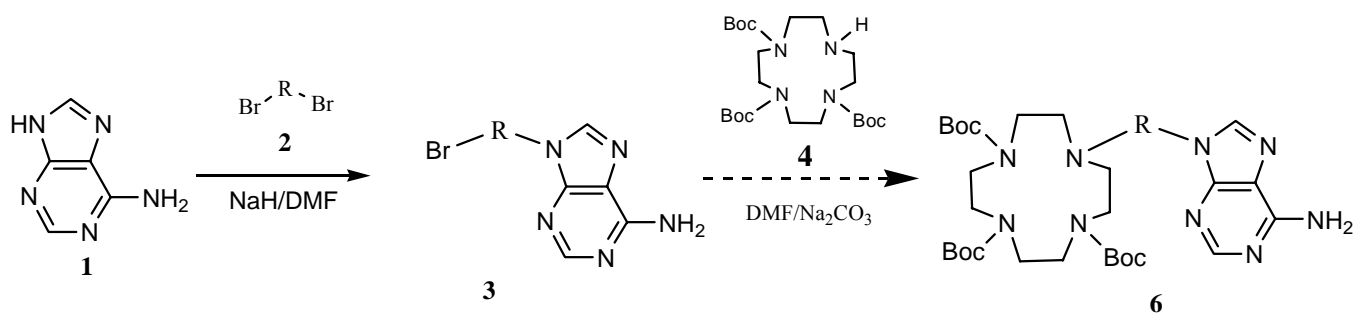

\section{Scheme 2}


Alternatively, we turned our attention to the second synthetic route which is shown in Scheme 3. This route was designed for the synthesis of conjugates under mild reaction conditions. The 1,4- and 1,3-bis(bromomethyl)benzenes $\mathbf{2 a}$ and $\mathbf{2 b}$ were conveniently coupled with (Boc) $)_{3}$ - cyclen 4 to produce compound 5 in the presence of sodium carbonate in about $70 \%$ yield. The linkage reaction between compound 5 and adenine were easily completed in DMF and in the presence of strong base $\mathrm{NaH}$. This reaction leads to the desired product $\mathbf{6}$ in high yields.
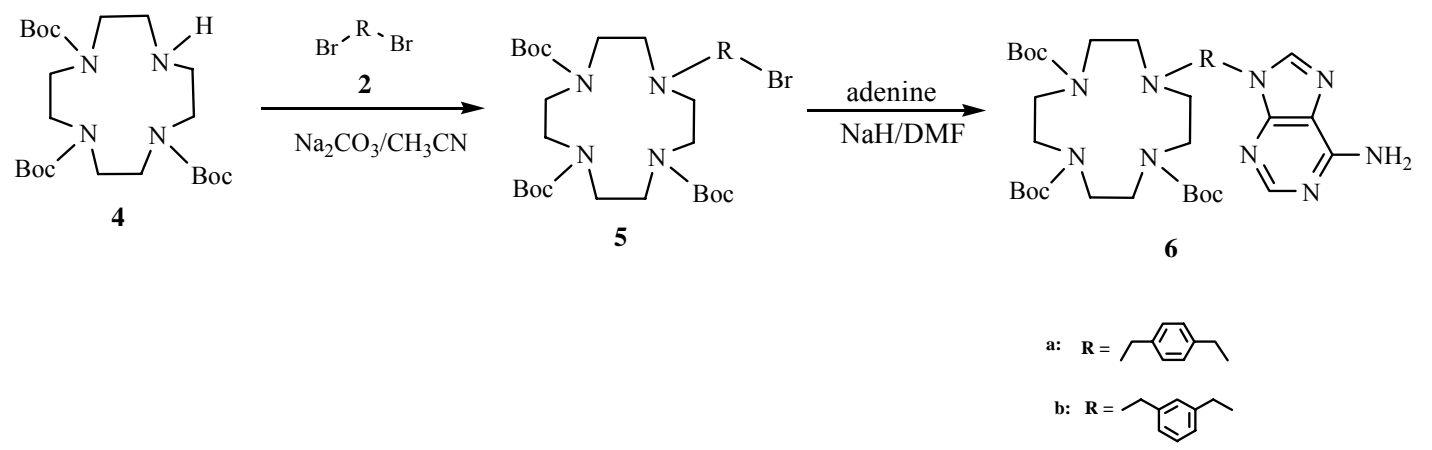

\section{Scheme 3}

As outlined in Scheme 4, removal of the Boc groups on 6 could be carried out using some strong acids, such as TFA, $\mathrm{HCl}, \mathrm{HBr}$, et al. Among these reagents, $47 \% \mathrm{HBr}$ enabled 7 to be easily precipitated in good shape after the complete deprotection and the reaction could be easily controlled. A yield of $78 \%$ was obtained with compound $7 \mathbf{a}$ and $73 \%$ for compound $7 \mathbf{b}$. In alkaline solution, preliminary results indicate that the affinity of adenine-cyclen conjugates towards transition metal ions such as $\mathrm{Zn}^{2+}$ is not affected by adenine and we could conveniently obtain the complexes $\mathbf{8}$ in high yields. Their catalytic feature for hydrolytic reactions is currently studied in our laboratory.

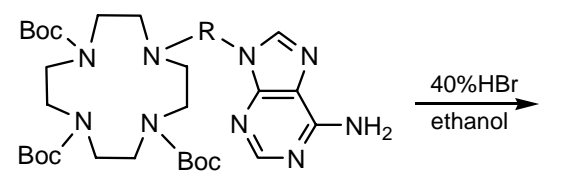

6

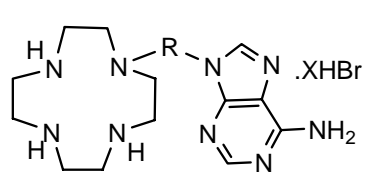

7

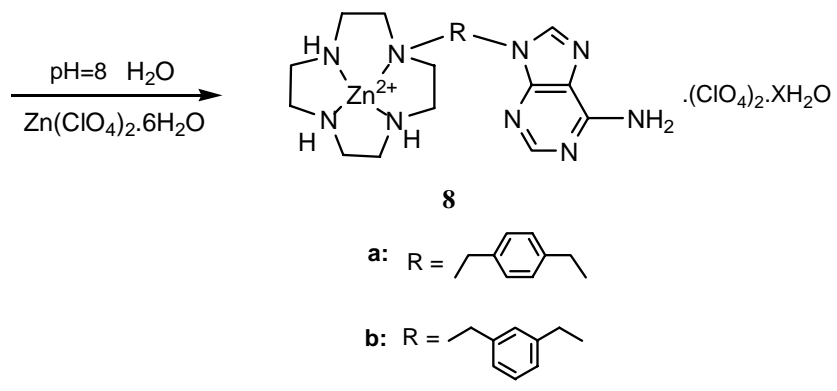

\section{Scheme 4}




\section{Experimental Section}

General Procedures. ESI-MS spectra data were recorded on Finnigan LCQ DECA mass spectrometer. ${ }^{1} \mathrm{H}$ NMR spectra were measured on a Varian INOVA-400 spectrometer and chemical shifts in ppm are reported relative to internal $\mathrm{Me} 4 \mathrm{Si}\left(\mathrm{CDCl}_{3}\right)$ or 3-(trimethylsilyl) propionic-2,2,3,3- $\mathrm{d}_{4}$ acid sodium salt $\left(\mathrm{D}_{2} \mathrm{O}\right)$. Melting points were determined by using a micromelting point apparatus without any corrections. 1, 4, 7-tris (tert-Butyloxycarbonyl)-1, 4, 7, 10tetraazacyclododecance (3Boc-cyclen) were prepared according to literature. ${ }^{23}$ Chemicals and reagents were obtained commercially and used without further purification.

1-[4, 7, 10-Tris(tert-butyloxycarbonyl)]-1, 4, 7, 10-tetraazacyclododecane (4). A solution of cyclen $(1,4,7,10$-tetraazacyclododecane) $(1.0 \mathrm{~g}, 5.8 \mathrm{mmol})$ and triethylamine $(2.5 \mathrm{~mL})$ in $40 \mathrm{~mL}$ of dry $\left.\mathrm{CHCl}_{3}\right)$ was stirred at room temperature, $(\mathrm{Boc})_{2} \mathrm{O}(3.8 \mathrm{~g}, 17.6 \mathrm{mmol})$ was added slowly to the mixture, then the reaction was stirred at room temperature for $72 \mathrm{~h}$. and the solvent was evaporated in vacuo. The residue was dissolved in $\mathrm{CHCl}_{3}$ and then purified by silica gel column chromatography (ethyl acetate: petroleum ether=3:1). A colorless amorphous solid 4 was obtained in $68.9 \%$ yield. Mp: $69-71{ }^{\circ} \mathrm{C}$. ESI-MS: $\mathrm{m} / \mathrm{z}=473.2(\mathrm{M}+\mathrm{H})^{+}$.

1-\{1-[4, 7, 10-Tris(tert-butyloxycarbonyl)]-1, 4, 7, 10-tetraazacyclododecane $\}$ methyl -4benzyl bromine (5a). Under a $\mathrm{N}_{2}$ atmosphere, a solution of 1, 4-bis(bromomethyl)benzene (1.03g, $4.00 \mathrm{mmol})$ and $\mathrm{Na}_{2} \mathrm{CO}_{3}(0.25 \mathrm{~g}, 2.17 \mathrm{mmol})$ in $50 \mathrm{~mL}$ of dry $\mathrm{CH}_{3} \mathrm{CN}$ was stirred. 1, 4, 7Tris (tert-butyloxycarbonyl)-1, 4, 7, 10-tetraazacyclododecane $4(0.50 \mathrm{~g}, 1.06 \mathrm{mmol})$ was added slowly to the mixture, and then the reaction was stirred at $80{ }^{\circ} \mathrm{C}$ for $72 \mathrm{~h}$. The insoluble inorganic salts were filtered off and the filtrate was concentrated in vacuo. The residue was dissolved in $\mathrm{CHCl}_{3}$ and then purified by silica gel column chromatography (ethyl acetate: petroleum ether=1:4). A colorless amorphous solid 5a was obtained in 68.9\% yield. Mp: $69-71{ }^{\circ} \mathrm{C}$. ESIMS: $\mathrm{m} / \mathrm{z}=757.5(\mathrm{M}+\mathrm{Na})^{+}$.

1-\{1-[4, 7, 10-Tris(tert-butyloxycarbonyl)]-1, 4, 7, 10-tetraazacyclododecane\} methylene-3benzyl bromine (5b). With the above-described synthetic method we could obtain compound 5b: Colorless amorphous solid, yield: 73.5\%. Eluent: EtOAc: petroleum ether=1: 4. Mp: 83$84{ }^{\circ} \mathrm{C}$. ESI-MS: $\mathrm{m} / \mathrm{z}=766.3\left(\mathrm{M}+\mathrm{Na}^{+}\right)$.

1-\{1-[4, 7, 10-Tris(tert-butyloxycarbonyl)]-1, 4, 7, 10-tetraazacyclododecane\} methylene -4(9-adenine) methylene benzene (6a). $\mathrm{NaH}(40 \mathrm{mg}, 1 \mathrm{mmol})$ was added to a suspension of adenine (2.2 mmol, $297.3 \mathrm{mg}$ ) and KI (60 mg, a catalytic amount) in dry, degassed N, Ndimethylformamide and stirred for $0.5 \mathrm{~h}$ at room temperature. A solution of $5 \mathrm{a}$ ( $2 \mathrm{mmol}, 1.30 \mathrm{~g})$ in dry DMF was added slowly to this mixture and the reaction was then stirred under $\mathrm{N}_{2}$ at $50{ }^{\circ} \mathrm{C}$ overnight. Water was added and the reaction mixture was extracted with ethyl acetate. The organic layer was dried with anhydrous $\mathrm{Na}_{2} \mathrm{SO}_{4}$ and concentrated in vacuo below $40{ }^{\circ} \mathrm{C}$ to give a crude product. The resulting crude product was purified by silica gel chromatography (methanol: chloroform $=1: 8)$ to afford colorless amorphous solid 6a (yield: $80 \%)$. Mp: $73-75{ }^{\circ} \mathrm{C}$. IR $(\mathrm{KBr}$ pellet): $3367,3325,3270,3166,2976,2930,1686,1598,1458,1414,1458,1414,1364,1250$, 
$1164,1105,977,858,774 \mathrm{~cm}^{-1} .{ }^{1} \mathrm{H} \mathrm{NMR}\left(\mathrm{CDCl}_{3}, \delta, 400 \mathrm{MHz}\right): 1.45\left(\mathrm{~s}, 27 \mathrm{H}, \mathrm{OC}\left(\mathrm{CH}_{3}\right)_{3}\right), 2.61-$ 2.64 (m, 2H, Ph- $\mathrm{CH}_{2}$-cyclen), 3.38-3.76 (m, $16 \mathrm{H}, \mathrm{CH}_{2} \mathrm{NCH}_{2}$ ), 5.32 (s, 2H, Ph- $\mathrm{CH}_{2}$-adenine), $5.85\left(\mathrm{~s}, 2 \mathrm{H}\right.$, adenine- $\left.\mathrm{NH}_{2}\right), 7.12-7.30(\mathrm{~m}, 4 \mathrm{H}, \mathrm{Ph}-\mathrm{H}), 7.74(\mathrm{~s}, 1 \mathrm{H}, \mathrm{H}(2)$ in adenine $), 8.37(\mathrm{~s}, 1 \mathrm{H}$, $\mathrm{H}(8)$ in adenine ); ESI-MS: $\mathrm{m} / \mathrm{z}=710.3(\mathrm{M}+\mathrm{H})^{+}$; HRMS (ESI) calcd. for $\mathrm{C}_{36} \mathrm{H}_{55} \mathrm{~N}_{9} \mathrm{O}_{6} \mathrm{Na}$ $[\mathrm{M}+\mathrm{Na}]^{+}: \mathrm{m} / \mathrm{z}=732.4168$. Found: 732.4202 .

1-\{1-[4, 7, 10-Tris(tert-butyloxycarbonyl)]-1, 4, 7, 10-tetraazacyclododecane\} methylene -3(9-adenine)methylene benzene (6b). With the above-described synthetic method we could obtain compound 6b: Colorless amorphous solid, yield: $78.7 \%$. Mp: $64-66{ }^{\circ} \mathrm{C}$, IR (KBr pellet): 3111, 3039, 2988, 2940, 2824, 2740, 1716, 1659, 1417, 1235, 845, 543, $436 \mathrm{~cm}^{-1}$. ${ }^{1} \mathrm{H}$ NMR $\left(\mathrm{CDCl}_{3}, \delta, 400 \mathrm{MHz}\right): 1.47\left(\mathrm{~s}, 27 \mathrm{H}, \mathrm{OC}\left(\mathrm{CH}_{3}\right)_{3}\right), 2.61-2.63\left(\mathrm{~m}, 2 \mathrm{H}, \mathrm{Ph}-\mathrm{CH}_{2}\right.$-cyclen), 3.23-3.71 $\left(\mathrm{m}, 16 \mathrm{H}, \mathrm{CH}_{2} \mathrm{NCH}_{2}\right.$ ), 5.34 (s, 2H, Ph- $\mathrm{CH}_{2}$-adenine), 5.72 (s, 2H, adenine- $\mathrm{NH}_{2}$ ). 7.18-7.30 (m, 4H, Ph-H), $7.64(\mathrm{~s}, 1 \mathrm{H}, \mathrm{H}(2)$ in adenine), $8.38(\mathrm{~s}, 1 \mathrm{H}, \mathrm{H}(8)$ in adenine $)$. ESI-MS: $\mathrm{m} / \mathrm{z}=710.3$ $\left(\mathrm{M}+\mathrm{H}^{+}\right)$. HRMS (ESI) calcd. For $\mathrm{C}_{36} \mathrm{H}_{55} \mathrm{~N}_{9} \mathrm{O}_{6} \mathrm{Na}[\mathrm{M}+\mathrm{Na}]^{+}: \mathrm{m} / \mathrm{z}=732.4275$. Found: 732.4208 .

1-(1,4,7,10-Tetraazacyclododecane)methylene-4-(9-adenine)methylenebenzene tetrahydrobromide (7a). To a solution of 6a $(0.709 \mathrm{~g}, 1.0 \mathrm{mmol})$ in dry $\mathrm{EtOH}(5 \mathrm{~mL})$ at $0{ }^{\circ} \mathrm{C}$, $40 \%$ aqueous $\mathrm{HBr}(1 \mathrm{~mL})$ was added slowly. After being stirred overnight at room temperature, the reaction mixture was concentrated in vacuo below $40{ }^{\circ} \mathrm{C}$ to give a crude product. The resulting crude product was recrystallized from $\mathrm{EtOH} / 24 \%$ aqueous $\mathrm{HBr}$ to afford $7 \mathbf{a}$ as white powder solid (yield, 78\%). Mp: 236-238 ${ }^{\circ} \mathrm{C} .{ }^{1} \mathrm{H}$ NMR $\left(\mathrm{D}_{2} \mathrm{O}, \delta, 400 \mathrm{MHz}\right): 2.94-3.25$ (m, 16H, $\mathrm{NCH}_{2} \mathrm{CH}_{2} \mathrm{~N}$ ), 3.66-3.72 (m, 2H, Ph- $\mathrm{CH}_{2}$-cyclen), 3.85-3.89 (m, 2H, Ph- $\mathrm{CH}_{2}$-adenine), 7.35-7.53 $(\mathrm{m}, 6 \mathrm{H}, \mathrm{PhH}$, adenine- $\mathrm{CH}) .8 .60(\mathrm{~s}, 1 \mathrm{H}, \mathrm{H}(2)$ in adenine), $8.67(\mathrm{~s}, 1 \mathrm{H}, \mathrm{H}(8)$ in adenine).ESI$\mathrm{MS}(\mathrm{m} / \mathrm{z}): 410.4\left(\mathrm{M}^{+}+1-4 \mathrm{HBr}\right)$. HRMS (ESI) calcd. For $\mathrm{C}_{21} \mathrm{H}_{32} \mathrm{~N}_{9}[\mathrm{M}+\mathrm{H}-4 \mathrm{HBr}]^{+}: \mathrm{m} / \mathrm{z}=$ 410.2757. Found: 410.2702 .

\section{1-(1,4,7,10-Tetraazacyclododecane)methylene-3-(9-adenine)methylenebenzene}

tetrahydrobromide (7b). White powder solid was obtained (yield 73.3\%). Mp: $190-191{ }^{\circ} \mathrm{C} .{ }^{1} \mathrm{H}$ NMR (DMSO, $\delta, 400 \mathrm{MHz})$ : 2.71-3.17 (m, 16H, $\left.\mathrm{CH}_{2} \mathrm{NHCH}_{2}\right), 3.75$ (s, 2H, Ph- $\mathrm{CH}_{2}$-adenine), 5.05 (br, $\left.4 \mathrm{H}, \mathrm{CH}_{2} \mathrm{NHCH}_{2}\right), 5.51$ (s, 2H, adenine- $\left.\mathrm{NH}_{2}\right), 7.34-7.38(\mathrm{~m}, 4 \mathrm{H}, \mathrm{PhH}) .8 .70(\mathrm{~s}, 1 \mathrm{H}, \mathrm{H}$ (2) in adenine), $8.75(\mathrm{~s}, 1 \mathrm{H}, \mathrm{H}(8)$ in adenine $)$. ESI - MS (m/z): $410.2\left(\mathrm{M}^{+}+1-4 \mathrm{HBr}\right)$. HRMS (ESI) calcd. For $\mathrm{C}_{21} \mathrm{H}_{32} \mathrm{~N}_{9}[\mathrm{M}+\mathrm{H}-4 \mathrm{HBr}]^{+}: \mathrm{m} / \mathrm{z}=410.2777$. Found: 410.2702.

\section{1-(1,4,7,10-Tetraazacyclododecane)methyl-4-(9-adenine)methylbenzenezinc(II)}

diperchlorate (8a). The hydrobromide salt 7a $(0.729 \mathrm{~g}, 1.0 \mathrm{mmol})$ was dissolved in $5 \mathrm{~mL}$ of water. After adjusted aqueous solution to alkaline $(\mathrm{pH} \geq 8), \mathrm{Zn}\left(\mathrm{ClO}_{4}\right)_{2} \bullet 6 \mathrm{H}_{2} \mathrm{O}(0.408 \mathrm{~g}$, $1.1 \mathrm{mmol})$ in EtOH $(5 \mathrm{~mL})$ was added. The mixture was stirred overnight at room temperate. The solution was gradually concentrated to give a white powder solid $8 \mathrm{a}$ in $88.3 \%$ yield. $\mathrm{Mp}>300{ }^{\circ} \mathrm{C}$. ${ }^{1} \mathrm{H}$ NMR (D $\left.{ }_{2} \mathrm{O}, \delta, 400 \mathrm{MHz}\right):$ 2.66-3.21 (m, 18H), 5.52 (s, 2H, adenine- $\left.\mathrm{CH}_{2}-\mathrm{Ph}\right), 7.36-7.37(\mathrm{~m}$, $4 \mathrm{H}, \mathrm{Ph}-\mathrm{H}), 7.78(\mathrm{~s}, 1 \mathrm{H}, \mathrm{H}(2)$ in adenine $), 8.29(\mathrm{~s}, 1 \mathrm{H}, \mathrm{H}(8)$ in adenine $)$, ESI-MS (m/z): 475 $\left(\mathrm{M}^{+}+1-2 \mathrm{ClO}_{4}-\mathrm{H}_{2} \mathrm{O}\right)$.

1-(1, 4, 7, 10-Tetraazacyclododecane)methylene -3- (9-adenine)methylene benzene zinc (II) diperchlorate (8b). White powder solid was obtained (yield 88.5\%), Mp>300 ${ }^{\circ} \mathrm{C},{ }^{1} \mathrm{H}$ NMR $\left(\mathrm{D}_{2} \mathrm{O}, \delta, 400 \mathrm{MHz}\right): 2.55-2.71\left(\mathrm{~m}, 18 \mathrm{H}, \mathrm{CH}_{2} \mathrm{NCH}_{2}\right), 5.37\left(\mathrm{~s}, 2 \mathrm{H}\right.$, adenine- $\left.\mathrm{CH}_{2}\right)$, 7.84-7.91 (m, 
4H, Ph-H), 8.04 (s, 1H, adenine-2-H), 8.13 (s, 1H, adenine-8-H). ESI-MS (m/z): $487\left(\mathrm{M}^{+}-2-\right.$ $\left.2 \mathrm{ClO}_{4}\right)$.

\section{Conclusions}

In conclusion, we have designed and synthesized the conjugates of adenine-cyclen which could combine with $\mathrm{Zn}^{2+}$. It may be useful for recognition and separation of uracil rich oligonucleotides in water solvents and may find application in the selective transport of uracil rich nucleotides across the biological membranes. Furthermore, this work can be extended to design receptors for the site-specific binding of nucleic acids.

\section{Acknowledgements}

This work was financially supported by the National Natural Science Foundation of China (No: 20132020, 20372051, 20471038), Program for New Century Excellent Talents in University, Specialized Research Fund for the Doctoral Program of Higher Education and Scientific Fund of Sichuan Province for Outstanding Young Scientist.

\section{References}

1. Davis, J. T. Angew. Chem. Int. Ed. 2004, 43, 668.

2. Sessler, J. L.; Kral, V.; Shishkanova, T. V.; Gale. P. A. Proc. Natl. Acad. Sci. USA. 2002, 99, 4848.

3. (a) Seeman, N. C. Chem. Biol. 2003, 10, 1151, (b) Salas, M.; Gordillo, B.; Gonzalez, F. J. ARKIVOC 2003, (xi), 72. (c) Nair, V.; Jeon, G.-S. ARKIVOC 2004, (xiv), 133. (d) Nair, V.; Chun, B.-K. ARKIVOC 2003, (i), 9.

4. (a) Thibault, R. J.; Hotchkiss, P. J.; Gray, M. ; Rotello, V. M. J. Am. Chem. Soc. 2003, 125, 11249. (b) Chiacchio, U.; Corsaro, A.; Iannazzo, D.; Piperno, A.; Pistarà, V.; Procopio, A.; Rescifina, A.; Romeo, G.; Romeo, R.; Siciliano, M. C. R.; Valveric, E. ARKIVOC 2002, (xi), 159.

5. (a) Binder, W. H.; Kunz, M. J.; Ingolic, E.; J. Polym. Sci: Part A: Polym. Chem. 2004, 42, 162. (b) Binder, W. H.; Kunz, M. J.; Kluger, C.; Hayn, G.; Saf, R. Macromolecules. 2004, 37, 1749 .

6. Rai, R.; Khatri, V. K.; Pandey, P. S. Supramol. Chem. 2004, 16, 581.

7. Lee, A. H. F.; Kool, E. T. J. Am. Chem. Soc. 2005, 127, 3332.

8. Sessler, J. L.; Jayawickramarajah, J.; Sherman, C. L.; Brodbelt, J. S. J. Am. Chem. Soc. 2004, 126, 11460. 
9. Alexander, V. Chem. Rev. 1995, 95, 273.

10. Caravan, P.; Ellison, J. J.; McMurry, T. J.; Lauffer, R. B. Chem. Rev. 1999, 99, 2293.

11. Bianchi, A.; Calabi, L.; Corana, F.; Fontana, S.; Losi, P.; Maiocchi, A.; Paleari, L.;

Valtancoli, B. Coord. Chem. Rev. 2000, 204, 309.

12. Reany, O.; Gunnlaugsson, T.; Parker, D. Chem. Commun. 2000, 473.

13. Lim, N. C.; Yao, L. L.; Freake, H.; Bruckner, C. Bioorg. Med. Chem. Lett. 2003, 13, 2251.

14. Shionoya, M.; Kimura, E.; Shiro, M. J. Am. Chem. Soc. 1993, 115, 6730.

15. Shionoya, M.; Ikeda, T.; Kimura, E.; Shiro, M. J. Am. Chem. Soc. 1994, 116, 3848.

16. Epstein, D. M.; Chappell, L. L.; Khalili, H.; Supkowski, R. M.; Horrocks, W. D. Jr.; Morrow, J. R. Inorg. Chem. 2000, 39, 2130.

17. Kimura, E.; Shionoya, M.; Hoshino, A.; Ikeda, T.; Yamada, Y. J. Am. Chem. Soc. 1992, 114, 10134.

18. Zhang, X.; van Eldik, R. Inorg. Chem. 1995, 34, 5606.

19. Brechbiel, M.; Pippin, C. G.; McMurry, T. J.; Milenic, D.; Roselli, M.; Colcher, D.; Gansow, O. A. J. Chem. Soc., Chem. Commun. 1991, 1169.

20. Kumar, K.; Magerstadt, M.; Gansow, O. A. J. Chem. Soc., Chem. Commun. 1989, 145.

21. Kimura, E.; Kitamura; Koike, T.; Shiro, M. J. Chem. Soc. 1997, 269, 102.

22. Atkins, T. J.; Richman, J. E.; Oettle, W. F. Org. Synth. 1978, 58, 86.

23. Kimura, E. Aoki, S. T. Koike, T.M. Shiro, M. J. Am. Chem. Soc. 1997, 119, 3068.

24. Kimura, E.; Aoki, S.; Koike, T.; Shiro, M. J. Am. Chem. Soc. 1997, 119, 3068. 